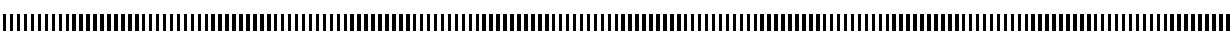



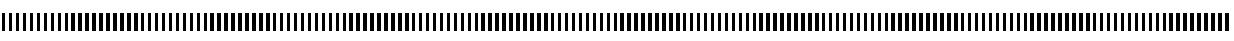

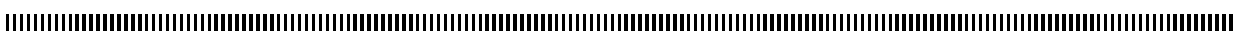
| | |

ARIMA Journal

\title{
Two Short Presentations related to Cancer Modeling
}

\author{
A. Habbal - P.-E. Jabin \\ Laboratoire de Mathématiques J.A.Dieudonné, \\ Université de Nice Sophia-Antipolis, \\ Parc Valrose, 06108 Nice Cedex 2, France
}

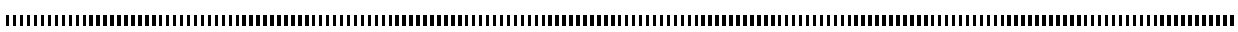

RÉSUMÉ. Dans ce papier, nous présentons deux modèles mathématiques du cancer. Le premier s'intéresse aux interactions entre tumeur et système immunitaire, qui interviennent lors des premières phases de développement des tumeurs cancéreuses. Celles-ci sont en compétition avec le système immun. On étudie les deux cas conduisant soit à l'élimination des cellules tumorales, soit à la viabilité d'une tumeur solide. Dans la seconde partie, on développe un modèle d'initiation d'angiogenèse dans une tumeur solide. Ce processus, qui munit la tumeur d'un système circulatoire propre est modélisé via la théorie des jeux, les joueurs étant les activateurs et les inhibiteurs du processus angiogénique.

ABSTRACT. This paper contains two short presentations related to the mathematical modeling of Cancer. The first part intends to introduce a tumour-immune system interaction, which describes the early dynamics of cancerous cells, competing with the immune system, potentially leading to either the elimination of tumoral cells or to the viability of a solid tumor. The second part of the paper addresses the case where a solid tumor has grown enough to initiate angiogenesis, a process which equips the tumor with its own blood network. Nash game theory is used to model the interaction between activators and inhibitors of the angiogenesis process.

MOTS-CLÉS : tumeur, cellules immunes, compétition, angiogenèse, jeux de Nash, optimisation topologique

KEYWORDS : tumor, immune cells, competition, angiogenesis, Nash games, topology optimization

| 


\section{A model for tumor-immune system competition}

This part is a summary of the results obtained in [13] [11] [12] and [14]. Those articles derive and study a class of models for the early stage of tumor development when tumor cells are not yet aggregated into a solid form. They may also be applied when cells are residually dispersed in the environment after the removal of a solid form.

The spatial distribution of tumor cells is, at this stage, not overly important and the models are therefore homogeneous in space. However it is essential to take the activity or progression of the tumor cells into account as it is affected by the immune system and the quantity of food available (which is in limited supply as angiogenesis did not occur yet).

The models also consider the activity of immune cells and consequently require the introduction of the two densities $f_{1}(t, u)$ and $f_{2}(t, u)$. The function $f_{1}$ is the density of tumor cells at time $t$ with activity $u \in \mathbb{R}^{+}$whereas $f_{2}(t, u)$ is the density of immune cells. The two biologically important quantities are the total numbers $n_{1}(t)$ and $n_{2}(t)$ of tumor and immune cells, which are defined as

$$
n_{i}(t)=\int_{\mathbb{R}^{+}} f_{i}(t, u) d u, \quad i=1,2 .
$$

However the important quantities for the evolution of the system are the total activities $A_{1}(t)$ and $A_{2}(t)$ given by

$$
A_{i}(t)=\int_{\mathbb{R}^{+}} u f_{i}(t, u) d u, \quad i=1,2 .
$$

Notice that the $A_{i}$ are in general not accessible to measure (one can typically only count the cells and not determine whether they are quiescent or not). The last important quantity is the quantity of food available in the environment which we simply denote by $A_{3}(t)$.

The interaction between cells are the following

- The interaction between a tumor cell and a immune cell decreases the activity of both cells ;

- Immune cells may kill or destroy tumor cells ;

- The presence of tumor cells induces the proliferation of immune cells ;

- The quantity of food available is depleted by the tumor cells but that increases their activity.

With these assumptions, the equation for the tumor cells simply reads

$$
\partial_{t} f_{1}+\partial_{u}\left(\left(\alpha_{13} A_{3}-\alpha_{12} A_{2}\right) u f_{1}\right)=\beta_{13} u A_{3} f_{1}-\beta_{12} A_{2} f_{2},
$$

whereas the equation for the immmune system is

$$
\partial_{t} f_{2}-\partial_{u}\left(\alpha_{21} A_{1} u f_{2}\right)=\beta_{21} A_{1} u f_{2},
$$

and for the environment we simply have

$$
\frac{d}{d t} A_{3}=-\gamma A_{1} A_{3}
$$

The system was derived in a more general framework in [13] and of course requires initial conditions

$$
f_{i}(t=0, u)=f_{i}^{0}(u) \in L^{1}((1+u) d u), \quad A_{3}(0)=A_{3}^{0} \in \mathbb{R}_{+} .
$$


The equations are weakly coupled through the total activities. This weak non linearity does not pose problems to obtain solutions but the control on the total activities is more delicate. Indeed any bound on a moment of the form $\int u^{k} f_{i}(t, u) d u$ would require a bound on the next moment $\int u^{k+1} f_{i}(t, u) d u$. So existence of solutions was obtained only in [11] under the conditions that

$$
\int_{\mathbb{R}_{+}} e^{\lambda u} f_{1}^{0}(u) d u<\infty, \forall \lambda>0 ; \quad \int_{\mathbb{R}_{+}} e^{\beta_{21} u / \alpha_{21}} f_{2}^{0}(u) d u<\infty .
$$

They may appear rather strong but are in fact somewhat optimal as it was proved in the same paper: No solution may exist, even for a short time, without some exponential decay on the initial data.

More interesting than the usual mathematical question of well-posedness is the study of the asymptotic behaviour in time. For this first model, the behaviour is very straightforward as either the tumor cells win, in which case the activities of the immune system and environment eventually vanish; Or the immune system wins, eliminating all tumor cells. More precisely, define

$$
n_{2}^{*}=\int_{\mathbb{R}_{+}} e^{\beta_{21} u / \alpha_{21}} f_{2}(t, u) d u,
$$

Then this number is conserved in time $\left(d n_{2}^{*} / d t=0\right)$. It is therefore the maximal number of immune cells $n_{2}(t)$ and we have either of the two

(i) $n_{1}(t) \longrightarrow 0, \quad \int_{0}^{\infty} A_{1}(t) d t<\infty, \quad n_{2}(t) \longrightarrow n_{2}(\infty)<\int_{0}^{\infty} e^{\beta_{21} u / \alpha_{21}} f_{2}^{0}(u) d u$, but $n_{3}, A_{3}, n_{2}, A_{2}$ are bounded from below.

(ii) $\int_{0}^{\infty} A_{1}(t) d t=\infty, n_{2}(t) \longrightarrow \int e^{\beta_{21} u / \alpha_{21}} f_{2}^{0}(u) d u, A_{2}(t), n_{3}(t), A_{3}(t) \longrightarrow 0$.

The model composed of (1.1), (1.2) and (1.3) is valid only for a short period of time during which no extra amount of food may be produced by the organism. If one wants to consider it for longer times, then some modifications have to be introduced, which is the object of [14]. For example (1.3) can be replaced by

$$
\frac{d}{d t} A_{3}=-\gamma A_{1} A_{3}+\delta\left(A_{3}^{*}-A_{3}\right) A_{3},
$$

where $A_{3}^{*}$ can represent the normal amount of nutriments and oxygen which are found in healthy tissues. The new term simply allows for the environment to recover to its normal healthy state in the absence of tumor cells.

The new system made of (1.1), (1.2) and (1.5) has the same asymptotic behaviour than the previous one. However it is also possible to allow for the immune system to recover, changing (1.2) into

$$
\partial_{t} f_{2}-\partial_{u}\left(\alpha_{21} A_{1} u f_{2}\right)=\beta_{21} A_{1} u f_{2}+\beta_{22}\left(f_{2}^{*}(u) A_{3}(t)-A_{3}^{*} f_{2}(t, u)\right) .
$$

The behaviour in large time is now much more complicated as the disease may become chronical (tumor cells lay low most of time but resurface again and again).

The biological interpretation of this mathematical analysis is already interesting as it predicts that the end of the disease will always be simple (death/tumor in solid form vs elimination of the tumor) only if it evolves fast enough with respect to the recovery time of the immune system.

Finally two therapeutical actions were introduced and studied in [12]. The first one acts on the environment to decrease the amount of available food for the tumor cells. Mathematically speaking we introduce a new quantity $A_{4}(t)$ satisfying

$$
\frac{d}{d t} A_{4}(t)=-\gamma_{4} A_{4} A_{3}
$$


and suitably modify the equation on $A_{3}$

$$
\frac{d}{d t} A_{3}(t)=-\gamma A_{1} A_{3}-\gamma_{3} A_{4} A_{3}
$$

The second one directly helps the immune system and, mathematically speaking, leads to the new equations

$$
\frac{d}{d t} A_{5}(t)=-\gamma_{5} A_{4} A_{2}
$$

and

$\partial_{t} f_{2}+\partial_{u}\left(\alpha_{25} A_{5} u f_{2}-\alpha_{21} A_{1} u f_{2}\right)=\beta_{21} A_{1} u f_{2}+\beta_{22}\left(f_{2}^{*}(u) A_{3}(t)-A_{3}^{*} f_{2}(t, u)\right)$.

In both cases $A_{4}$ and $A_{5}$ represent the treatment which is given to the patient all in once at time 0 .

The two treatments do not have the same effect. Whereas if $A_{5}(0)$ is large enough then the system always evolve toward the elimination of the tumor cells, this is not the case for the first treatment. There are initial values for $f_{1}^{0}$ and $f_{2}^{0}$ such that, no matter how high $A_{4}^{0}$ is, the tumor cells always win.

However this does not mean that either of the treatments is without risk. Namely, it was not possible to prove that their effect is never damageable: There could be initial data $f_{1}^{0}, f_{2}^{0}$ and $A_{3}^{0}$ for which the normal evolution is the elimination of the tumor cells whereas if we add a treatment (with small $A_{4}^{0}$ or $A_{5}^{0}$ ) the opposite happens.

This implies that the treatments should always be strong enough, which is a problem in real situations as they typically have side effects. Moreover, even though the second treatment is better according to the analysis, numerical simulations indicate that a combination of the two is much more effective : A positive outcome can be ensured with much lower values of $A_{4}^{0}$ and $A_{5}^{0}$.

\section{Tumoral Angiogenesis and anti-angiogenesis}

The results presented in this section are excerpt from the paper [19]. The reader is invited to refer to op. cit. for more details.

At their early stage of growth, solid tumors are avascular. They do not need a blood network, being small enough to get nutrients mainly by tissue diffusion.

However, their needs are proportional to their -growing- volume, while the feeding is proportional to the surface in contact with the host tissue. So, they rapidly reach a critical size for which the supply by diffusion is no more enough to continue developing. Then, avascular tumors sometimes turn into a dormant phase during which the growth stops, as a result of balance between proliferation and apoptosis -death- of cancer cells.

Tumors which do not enter dormancy need ways alternative to diffusion. It is now well known that solid tumors use vascular supply. Tumor-associated neovascularization allows the tumor cells to express their critical growth advantage as reported by Saaristo et al.[25]. The process by which solid tumors develop a vascular network is called angiogenesis. Angiogenesis is a complex process, a complete description of which is outside the scope of the present paper. Readers interested in fundamental basics, particularly in view of mathematical modeling could refer to the well documented review paper by Mantzaris, Webb and Othmer [22]. 


\subsection{Mathematical modeling}

It is likely that the first mathematical modeling of solid tumor growth amounts to the paper by Collins et al. [10] in 1956. A few papers were then dedicated to cancer related mathematics, less than one per year, until the early 1970s with the introduction of a diffusion model by Greenspan in 1972 [18]. Mathematical models based on diffusion gained then a large audience, with an increasing number of publications, about four or five per year, see the review by J. Adam in 1989 [1]. The computational effort was minimal, and consisted in producing curves of temporal or one dimensional spatial response, until the contribution by Duchting with 3D computations in 1989 [15]. Specific targetting of angiogenesis modeling as a part of tumor growth was first addressed by Chaplain and Sleeman in 1990 [8]. Since then, an important contribution to mathematical and 4D-computational tumoral angiogenesis was made by Chaplain, Sleeman, Anderson [2] [3]. For a more complete review, see Othmer [22] where an exhaustive bibliography is presented.

Most if not all of the above contributions use mathematical models of nonlinear parabolic reaction-diffusion type. These models are based on equations which express balance or conservation laws of physical relevant quantities like as blood cells or extracellular matrix densities. The full dynamics of the tumor growth are determined starting from given initial conditions. An illustrative presentation excerpt from Chaplain [2] is as follows :

$$
\begin{aligned}
& n_{t}=\overbrace{\nabla_{\nabla \cdot d_{n} \nabla n}^{\text {random motility }}-\overbrace{\chi \nabla \cdot\left(\frac{n}{k+c} \nabla c\right)}^{\text {chemotaxis }}-\overbrace{\rho \nabla \cdot n \nabla f}^{\text {haptotaxis }}}^{\text {production }} \overbrace{\overbrace{\text { un }}^{\text {uptake }}}^{\text {degradation }}-\overbrace{\mu n f}^{\overbrace{\lambda n c}}
\end{aligned}
$$

where

$n:$ density of the blood vessels

$f$ :density of the matrix tissue

$c$ : concentration of angiogenic factors

Obviously, solid tumor growth is not only a question of diffusion; it should and in fact it does include key factors from structural mechanics. Indeed, there are only a very few and quite recent contributions to this area. In Chaplain and Sleeman [9], elasticity theory is used to describe tumor invasion. Jones et al. in [21] introduce a constitutive law that combines the stress-strain relation of linear elasticity with a growth term derived by analogy with thermal expansion. Tumor spheroid growth is also studied with poroelasticity modeling in [23] [26]. A more recent study by Araujo and McElwain addressing growth-induced stresses in tumors can be found in [4], the model presented highlights the role of various tissue properties in inducing vascular collapse phenomena observed inside tumors.

Our aim is to define a framework well adapted to the formulation of angiogenesis and anti-angiogenesis as a theoretical Nash game.

We shall deal with rather classical linear elliptic partial differential equations, within a framework for which existence and uniqueness of solutions is well known, [17]. So, for the sake of clarity of the exposure, we do not detail standard functional spaces, weak formulations and regularity assumptions on the data, unless if necessary. 


\subsubsection{A porous media model for the tumor}

The extracellular matrix as well as the tumoral vasculature are seen as a porous medium, which occupies a volume $\Omega \subset \mathbb{R}^{N}(N=2$ or 3$)$, with a variable permeability denoted by $\rho$, which lies between the matrix permeability $\rho_{M}$ and blood vessel permeability $\rho_{V}$ :

$$
0 \leq \rho_{M} \leq \rho \leq \rho_{V}
$$

The simplest effective (or homogenized) model for porous media is the following, also known as the Darcy Law, where the physical unknown variable is pressure $p$ :

$$
\left\{\begin{array}{lrrr}
-\operatorname{div}(\rho \nabla p) & = & Q & \text { in } \Omega \\
\rho \frac{\partial p}{\partial n} & = & \rho g & \text { over } \Gamma_{V} \\
\frac{\partial p}{\partial n} & = & 0 & \text { over } \Gamma_{N} \\
p & = & 0 & \text { over } \Gamma_{T}
\end{array}\right.
$$

The right-hand side $Q$ represents a residual source of nutrients by diffusion through the host tissue, it is assumed to be negligible compared to the inward blood flow $g$. It should be noticed that we do not take into account what happens inside the tumor itself, considering only its boundary $\Gamma_{T}$ as an outlet.

Obviously, the pressure field depends on the permeability distribution.

We postulate that angiogenesis provides the tumor with an optimal drainage mechanism, i.e. with a permeability such that the tumor optimal blood network minimizes the averaged pressure drop.

The pressure drop denoted by $L_{1}(\rho ; p)$ is given by the formula :

$$
L_{1}(\rho ; p)=\int_{\Omega} Q p d x+\int_{\Gamma_{V}} \rho g p d s
$$

\subsubsection{A structural model for the extracellular matrix}

Now, one may also consider the host surrounding tissue as a continuum medium, let us say a linear isotropic, nonhomogeneous, elastic material. This model is of course a coarse approximation of the actual mechanical behavior of the living tissues, which is rather of visco-elastic nature [16]. This medium is composed of healthy and degraded tissues. The degradation could be due to established vascularization or to an early enzyme's action, like as the MMPs family.The elasticity tensor $\mathbf{E}$ lies then (in a certain sense) between the degraded material tensor $\mathbf{E}_{\mathbf{D}}$, and the original -sane- extracellular matrix tensor $\mathbf{E}_{\mathbf{M}}$.

Conforming to the linear elasticity classical equilibrium equations, the displacement vector $\mathbf{u}=\left(u_{j}\right)$ solves

$$
\left\{\begin{array}{lrrr}
-\operatorname{div}(\mathbf{E} \epsilon(\mathbf{u})) & = & \mathbf{b} & \operatorname{in} \Omega \\
\mathbf{u} & = & \mathbf{0} & \text { over } \Gamma_{V} \\
\mathbf{E} \epsilon(\mathbf{u}) \cdot \mathbf{n} & = & \mathbf{0} & \text { over } \Gamma_{N} \\
\mathbf{E} \epsilon(\mathbf{u}) \cdot \mathbf{n} & = & \mathbf{t} & \text { over } \Gamma_{T}
\end{array}\right.
$$


The strain tensor denoted by $\epsilon(u)$ is defined with obvious notations as

$$
\epsilon(\mathbf{u})_{i j}=\frac{1}{2}\left(\frac{\partial u_{i}}{\partial x_{j}}+\frac{\partial u_{j}}{\partial x_{i}}\right)
$$

The mechanical stress tensor is given by $\sigma(\mathbf{u})=\mathbf{E} \epsilon(\mathbf{u})$.

The body forces -such as selfweight- are denoted by $\mathbf{b}$, and the normal tension which models the stress induced by the tumor growth is denoted by $\mathbf{t}$. The tissue is assumed to be clamped to the mother vessel $\Gamma_{V}$. A related model can be found in [21] where the authors study the stress induced during avascular tumor growth.

The displacement vector $\mathbf{u}$ depends on the Elasticity tensor $\mathbf{E}$. The latter itself depends on the interaction between activators and inhibitors of tissue degradation.

We assume that the host tissue is willing to keep its integrity, by using all available factors it could control (one example is inhibitors of MMPs).

In continuum mechanics, it is usual to consider that such goal is achieved by maximizing the stiffness, or equivalently, minimizing the compliance :

$$
L_{2}(\mathbf{E} ; \mathbf{u})=\int_{\Omega} \mathbf{b} \cdot \mathbf{u} d x+\int_{\Gamma_{T}} \mathbf{t . u} d s
$$

\subsection{Mathematical formulation of the game}

It turns out that our approach naturally fits into the topology design framework, amongst a large literature, one could refer to [6] and to the references therein. Multidisciplinary topology design solved as Nash game can be found in [20].

We consider a two-players static game of complete information. The two players are the Tumoral Angiogenic Factors (TAF) which control activators distribution, denoted by $\mu$, and anti-Angiogenic Factors (aAF) which control inhibitors distribution, denoted by $k$.

Strategy spaces are defined as follows :

- (TAF) is equipped with a strategy space

$$
S_{1}=\left\{\mu \in L^{\infty}(\Omega), \quad 0 \leq \mu \leq 1, \quad \int_{\Omega} \mu d x \leq \gamma_{1}|\Omega|\right\}
$$

$-(\mathrm{aAF})$ is equipped with a strategy space

$$
S_{2}=\left\{k \in L^{\infty}(\Omega), \quad 0 \leq k \leq 1, \quad \int_{\Omega} k d x \leq \gamma_{2}|\Omega|\right\}
$$

The constraints on the relative volume fractions express the fact that there is only a limited available amount of activators and inhibitors.

A simultaneous (or blind) choice of $(\mu ; k)$ prompts an interaction between TAF and aAF, which is modeled as follows :

- Interaction Law : $\theta=\mu(1-k)$

- Permeability $: \rho=\rho(\mu ; k)=\rho_{M}+\left(\rho_{V}-\rho_{M}\right) P(\theta)$ 
- Elasticity tensor : $\mathbf{E}=\mathbf{E}(\mu ; k)=\mathbf{E}_{M}+\left(\mathbf{E}_{D}-\mathbf{E}_{M}\right) P(\theta)$

where $P(\theta)$ is the identity, an exact homogenization operator, or an interpolated SIMPlike (Solid Isotropic Material Penalization) operator, see Rozvany et al. [24].

The interaction law is a very simple, arbitrary choice. It states for example that the inhibitor action is completely and immediately efficient. Actual biological situations are of course much more complex.

>From other part, even if we content ourselves with linear porous media and elasticity models, there is a need for a more accurate effective fluid and structural equations, taking into account at least microscopic progressive degradation of the medium.

To end with the definition of the game, objective or loss functions are defined respectively as :

$$
\begin{aligned}
\text { Pressure Drop } & j_{1}(\mu ; k)=L_{1}(\rho ; p) \text { for player }(\mathrm{TAF}) \\
\text { Mechanical Compliance } & j_{2}(\mu ; k)=L_{2}(\mathbf{E} ; \mathbf{u}) \text { for player }(\mathrm{aAF})
\end{aligned}
$$

where $p$ is the pressure solution to the Darcy equation (2.12), and $\mathbf{u}$ is the displacement vector solution to the elasticity equation (2.13).

Let us finally remark that even if the original game considered here is a noncooperative static game, computational requirements lead us to consider iterative solving methods. The algorithmic version mimics then a repeated, partially cooperative game since the two players exchange information about their respective partial optima during the iterative process.

\section{Existence of a Nash equilibrium}

We consider the cases where either $P(\theta)=\theta$ or $P(\theta)$ is a restriction operator, i.e. $P(\theta)=g \circ S_{R}(\theta)$, with $g$ being a convex function and $S_{R}$ a linear compact filter, cf [7] for details. We have the

Theorem 2.1 There exists a Nash equilibrium $\left(\mu^{\star}, k^{\star}\right) \in S_{1} \times S_{2}$ such that

$$
\begin{array}{ll}
\mu^{\star} \text { solves } & \min _{\mu \in S_{1}} j_{1}\left(\mu, k^{\star}\right) \\
k^{\star} \text { solves } & \min _{k \in S_{2}} j_{2}\left(\mu^{\star}, k\right)
\end{array}
$$

Proof. Let us first notice that the strategy spaces $S_{1}$ and $S_{2}$ are convex and compact for the weak-star $L^{\infty}$ topology.

>From one part, in case of $P(\theta)=\theta$ and since the functions $j_{1}$ and $j_{2}$ are the respective compliances of Darcy and Elasticity equations, it is well known that these functions can be expressed as supremum envelops of continuous affine functions with respect to respectively $\mu$ and to $k$ (using a variational formulation of the equations (2.12) and (2.13)), so these functions are convex and weak-star lower semicontinuous.

>From other part, if $P(\theta)$ is a restriction operator, $j_{1}$ and $j_{2}$ can still be expressed as supremum envelops of continuous convex (but not necessarily affine) functions with respect to respectively $\mu$ and $k$. Convexity is preserved thanks to linearity of the filter, and 
to convexity of $g$. Compactness of the filter implies the weak-star lower semicontinuity of the objectives.

The assumptions are fulfilled in order to apply the Nash existence theorem,which yields the existence of a Nash equilibrium, see Aubin [5].

\section{A Numerical Experiment}

We use a classical finite element method in order to solve equations (2.12) and (2.13). First, density distributions $\mu$ and $k$ are approximated by means of piecewise-constant interpolation, over triangular three-nodes elements. Then, the pressure and the displacements are approximated by $P 1$-triangular elements. We use a gradient-based optimization method in order to solve, iteratively, each of the player's programs. The gradients with respect to the strategies of the respective players were computed by means of the adjoint method.

We present a case where the tumour is a circular hole located inside a trapezoid. The initial vessel is located on the upper side of the trapezoid. The game is a zero-sum variant, where activators aim to maximise the tumour drainage while the inhibitors play with exactly the opposite objective.

The numerical results presented in figures 1-2-3 illustrate how during the iterative solving of the Nash game activators try to optimally connect the vessel to the tumour, while inhibitors try to find an optimal location to prevent vessel-tumour connections.

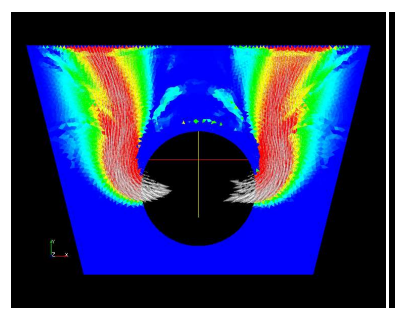

Figure 1. Activators.

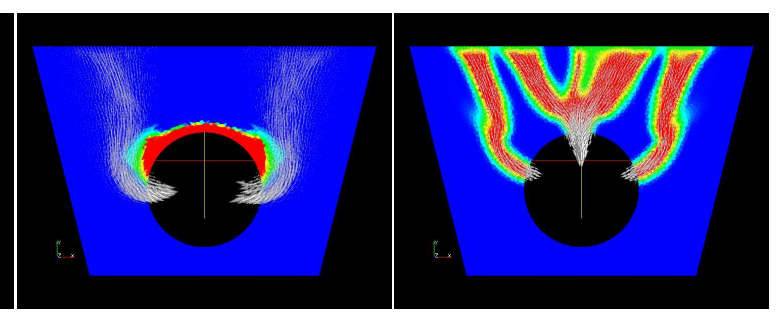

Figure 2. Inhibitors.

Figure 3. Final Network.

\section{Bibliographie}

[1] J.A. Adam. Diffusion models of prevascular and vascular tumor growth. A review. Lect. Notes Pure Appl. Math. , (131) :625-652, 1991.

[2] A.R.A. Anderson and M.A.J. Chaplain. Continuous and discrete mathematical models of tumour induced angiogenesis. Bull. Math. Biol., 60 :857-899, 1998.

[3] A.R.A. Anderson, M.A.J. Chaplain, C. Garcia-Reimbert, and C.A. Vargas. A gradient-driven mathematical model of antiangiogenesis. Math. Comput. Modelling, 32(10):1141-1152, 2000.

[4] R.P. Araujo and D.L.S. McElwain. New insights into vascular collapse and growth dynamics in solid tumors. Journal of Theoretical Biology, 228 :335-346, 2004.

[5] J.P. Aubin. Mathematical methods of game and economic theory. North-Holland Publishing Co. Amsterdam, New York, 1979.

[6] M.P. Bendsoe. Optimization of structural topology, shape, and material. Berlin : SpringerVerlag., 1995. 
28 A R I M A - Volume $10-2008 / 2009$

[7] T. Borrvall and J. Petersson. Topology optimization using regularized intermediate density control. Comput. Methods Appl. Mech. Eng., 190(37-38) :4911-4928, 2001.

[8] M.A.J. Chaplain and B.D. Sleeman. A mathematical model for the production and secretion of tumour angiogenesis factor in tumours. IMA J. Math. Appl. Med. Biol., 7(2) :93-108, 1990.

[9] M.A.J. Chaplain and B.D. Sleeman. Modelling the growth of solid tumours and incorporating a method for their classification using nonlinear elasticity theory. J. Math. Biol., 31(5) :431-473, 1993.

[10] V.P. Collins, R.K. Loeffler, and H. Tivey. Observations on growth rates of human tumors. Am J Roetgenol Radium Ther Nucl Med, 76 :988-1000, 1956.

[11] E. De Angelis and P.E. Jabin. Qualitative analysis of a mean field model of tumour-immune system competition. Math. Models Methods. Appl. Sci., 13 :187-206, 2003.

[12] E. De Angelis and P.E. Jabin. Mathematical models of therapeutical actions related to tumour and immune system competition. Math. Models Methods. Appl. Sci., 28(17) :2061-2083, 2005.

[13] E. De Angelis and L. Mesin. Modelling of the immune response : Conceptual frameworks and applications. Math. Models Methods Appl. Sci., 11 :1609-1630, 2001.

[14] L. Derbel. Analysis of a new model for tumor-immune system competition including long time scale effects. Math. Models Methods. Appl. Sci., 14 :1657-1681, 2004.

[15] W. Duchting. Recent progress in 3-d computer simulation of tumor growth and treatment. Acta Appl. Math., 14(1) :155-166, 1989.

[16] Y.C. Fung. Biomechanics. Mechanical properties of living tissues. 2nd ed. Springer-Verlag New York Berlin Heidelberg., 1993.

[17] D. Gilbarg and N.S. Trudinger. Elliptic Partial Differential Equations of Second Order. Springer-Verlag, Berlin Heidelberg NewYork, 1977.

[18] H.P. Greenspan. Models for the growth of a solid tumor by diffusion. Studies appl. Math., $51: 317-340,1972$.

[19] A. Habbal. A topology nash game for tumoral anti-angiogenesis. Journal of Structural Multidisciplinary Optimization, 30(5):404-412, November 2005.

[20] A. Habbal, J. Petersson, and M. Thellner. Multidisciplinary topology optimization solved as a nash game. Int. J. Numer. Methods in Eng., 61 :949-963, 2004.

[21] A.F. Jones, H.M. Byrne, J.S. Gibson, and J.W. Dold. A mathematical model of the stress induced during avascular tumour growth. J. Math. Biol., 40(6):473-499, 2000.

[22] N.V. Mantzaris, S. Webb, and H.G. Othmer. Mathematical modeling of tumor-induced angiogenesis. J. Math. Biol., 2004.

[23] T. Roose, P.A. Netti, L.L. Munn, Y. Boucher, and R.K. Jain. Solid stress generated by spheroid growth estimated using a linear poroelasticity model. Microvascular Research, 66 :204-212, 2003.

[24] G.I.N. Rozvany, M. Zhou, and T. Birker. Generalized shape optimization without homogenization. Struct Optim., 4 :250-254, 1992.

[25] A. Saaristo, T. Karpanen, and K. Alitalo. Mechanisms of angiogenesis and their use in the inhibition of tumor growth and metastasis. Oncogene, 19 :6122-6129, 2000.

[26] M. Sarntinoranont, F. Rooney, and M. Ferrari. Interstitial stress and fluid pressure within a growing tumor. Annals of Biomedical Engineering, 31 :327-335, 2003. 